\title{
Variational Formulation of Interaction between Elastic Plate and Elastic Medium under the Influence of Surface Energy
}

\author{
S. Tirapat ${ }^{1}$, T. Senjuntichai ${ }^{1}$, J. Rungamornrat ${ }^{1}$ and R. K. N. D. Rajapakse ${ }^{2}$ \\ ${ }^{1}$ Applied Mechanics and Structures Research Unit, Department of Civil Engineering, Faculty of Engineering, Chulalongkorn University, \\ Bangkok 10330 Thailand \\ ${ }^{2}$ School of Engineering Science, Simon Fraser University, Burnaby V5A 1 S6 Canada
}

\begin{abstract}
A variational formulation of interaction problem is presented in this paper for the analysis of an elastic circular plate under axisymmetric vertical loading resting on an isotropic elastic half-space under the influence of surface energy. The Gurtin-Murdoch surface elasticity theory is adopted to take into account the surface energy effects. The contact surface between the plate and the half-space is assumed to be smooth, and the deflected shape of the plate is represented by a power series of the radial coordinate. The undetermined coefficients in the series are determined through the minimization of the total potential energy functional of the plate-half-space system. The accuracy of the present solution is verified by comparing with existing solutions, and selected numerical results are presented to portray the influence of surface energy effects on interaction between an elastic circular plate and an elastic half-space.
\end{abstract}

\section{Introduction}

Nano-scale materials are found to possess excellent mechanical properties when compared to conventional engineering materials. Besides conducting experiments, analytical investigation is also performed to study mechanical behaviors at such small scale. Two analytical approaches have commonly been employed, namely atomistic simulations and modified continuum-based models. The first approach yields more accurate results but requires a lot more computational efforts and resources. The latter one then becomes an attractive choice in obtaining first-approximation to nano-scale systems. In this method, the surface energy effects have to be considered due to the high surface to volume ratio at nano-scale level. To take into account the influence of surface energy, Gurtin and Murdoch [1-2] presented a mathematical framework based on continuum mechanics concepts. In their model, the elastic surface is formed as an idealized layer of zero thickness perfectly bonded to the underlying bulk material without slipping. The surface elastic constants can be determined by atomistic and molecular static simulations [3], whereas the bulk material is governed by the classical elasticity theory. The Gurtin-Murdoch continuum-based model has been employed to study a variety of problems with the consideration of surface energy effects in the past (e.g. see [4-14]).

The present study is concerned with the analysis of interaction problem between a circular nano-plate of radius $a$ under axisymmetric vertical loading resting on an isotropic elastic half-space as shown in Figure 1 with the consideration of surface energy effects. This interaction problem is studied by employing a variational approach and the Gurtin-Murdoch surface elasticity theory [1-2]. The contact surface between the plate and half-space is assumed to be smooth, and the vertical displacement of the plate is represented by a power series of radial coordinate. The normal contact traction is expressed in terms of these generalized coordinates through the solutions of the flexibility equations based on the Green's functions for an elastic half-space under axisymmetric surface loading and surface energy influence, expressed in the form of Hankel integral transform [14]. Finally, the solution to the generalized coordinates can be obtained from the minimization of the total potential energy of the plate-half-space system. The accuracy of proposed solution scheme is confirmed through the comparison with exiting solutions. Selected numerical results for deflection and bending moment of the nano-plate are presented to demonstrate a significant influence of surface energy effects on the interaction problem.

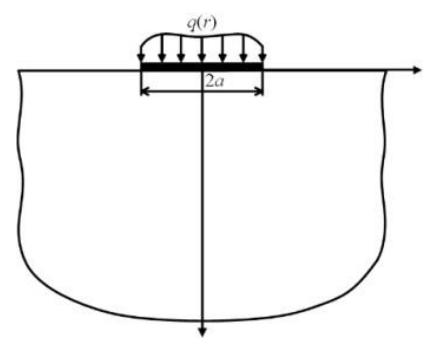

Figure 1. A circular nano-plate on an elastic half-space under axisymmetric vertical loading 


\section{Basic equations}

Consider an isotropic elastic half-space undergoing axisymmetric deformations with the cylindrical coordinate system $(r, \theta, z)$ and surface energy effects as shown in Figure 1. According to the Gurtin-Murdoch continuum model [1-2], the surface is a zero-thickness layer perfectly bonded to the bulk material without slipping. The basic equations for the bulk follow directly from the classical linear elasticity theory. On the surface, the equilibrium equation, the constitutive relations and the strain-displacement relationship are respectively expressed for axisymmetric deformations as,

$$
\begin{gathered}
\frac{\partial \sigma_{r r}^{s}}{\partial r}+\frac{\sigma_{r r}^{s}-\sigma_{\theta \theta}^{s}}{r}+\sigma_{z r}+t_{r}^{0}=0 ; \quad \frac{\partial \sigma_{z r}^{s}}{\partial r}+\frac{\sigma_{z r}^{s}}{r}+\sigma_{z z}+t_{z}^{0}=0 \\
\sigma_{r r}^{s}=\tau^{s}+\left(2 \mu^{s}+\lambda^{s}\right) \varepsilon_{r r}^{s}+\left(\lambda^{s}+\tau^{s}\right) \varepsilon_{\theta \theta}^{s} ; \sigma_{\theta \theta}^{s}=\tau^{s}+\left(2 \mu^{s}+\lambda^{s}\right) \varepsilon_{\theta \theta}^{s}+\left(\lambda^{s}+\tau^{s}\right) \varepsilon_{r r}^{s} ; \sigma_{z r}^{s}=\tau^{s} \frac{d u_{z}^{s}}{d r} \\
\varepsilon_{r r}^{s}=\frac{d u_{r}^{s}}{d r} ; \quad \varepsilon_{\theta \theta}^{s}=\frac{u_{r}^{s}}{r}
\end{gathered}
$$

where the superscript " $s$ " is used to denote the quantities corresponding to the surface (or interface); $\mu^{s}$ and $\lambda^{s}$ are surface Lamé constants; and $\tau^{s}$ is the residual surface stress (or surface tension) under unstrained condition. In addition, $t_{i}^{0}$ denotes the prescribed surface traction in the $i$-direction $(i=r, z)$.

\section{Variational formulation of interaction problem}

\subsection{Potential energy of elastic circular nano-plate}

Consider an elastic circular nano-plate of radius $a$ subjected to axisymmetric vertical loading as shown in Figure 1 . The deflection of the plate, denoted by $w(r)$, can be represented in the following form:

$w(r)=\sum_{n=0}^{N} \alpha_{n} r^{2 n}, \quad 0 \leq r \leq a$

where $\alpha_{n}(n=0,1, \ldots, N)$ denotes a set of generalized coordinates.

In the classical thin plate theory, the out of plane stress $\sigma_{z z}$ is neglected. According to Lim and He [15]; and Lu et al. [16], $\sigma_{z z}$ is assumed to vary linearly through the plate thickness to satisfy the equilibrium conditions along the interface, and $\sigma_{z z}$ can then be expressed as,

$\sigma_{z z}=\frac{1}{2}\left(\sigma_{z z}^{+}+\sigma_{z z}^{-}\right)+\frac{z}{h}\left(\sigma_{z z}^{+}-\sigma_{z z}^{-}\right)=\frac{2 \tau_{P}^{s} z}{h}\left(\frac{d^{2} w}{d r^{2}}+\frac{1}{r} \frac{d w}{d r}\right)$

where $\sigma_{z z}^{+}$and $\sigma_{z z}^{-}$are the stresses at the top and bottom surfaces respectively of the bulk of the plate; $h$ is the plate thickness; and $\tau_{P}^{s}$ is the residual surface stress (or surface tension) under unstrained condition of the plate. In addition, the other normal stresses in terms of generalized displacement, $w(r)$, can be expressed as,

$\sigma_{r r}=\frac{E}{1+v}\left[\frac{1}{1-v}\left(-z \frac{d^{2} w}{d r^{2}}\right)+\frac{v}{1-v}\left(-\frac{z}{r} \frac{d w}{d r}\right)\right]+\frac{v}{1-v} \frac{2 \tau_{P}^{s} z}{h}\left(\frac{d^{2} w}{d r^{2}}+\frac{1}{r} \frac{d w}{d r}\right)$ $\sigma_{\theta \theta}=\frac{E}{1+v}\left[\frac{1}{1-v}\left(-\frac{z}{r} \frac{d w}{d r}\right)+\frac{v}{1-v}\left(-z \frac{d^{2} w}{d r^{2}}\right)\right]+\frac{v}{1-v} \frac{2 \tau_{P}^{s} z}{h}\left(\frac{d^{2} w}{d r^{2}}+\frac{1}{r} \frac{d w}{d r}\right)$

where $E$ and $v$ is the Young's modulus and Poisson's ratio of the plate.

The strain energy of the plate contains two parts, i.e., the elastic strain energy stored in the bulk $\left(U_{B}\right)$ and the elastic strain energy of the surface $\left(U_{S}\right)$, which are expressed respectively as,

$U_{B}=\frac{1}{2} \int_{V}\left(\sigma_{r r} \varepsilon_{r r}+\sigma_{\theta \theta} \varepsilon_{\theta \theta}\right) d V$
$U_{S}=\frac{1}{2} \int_{\Gamma}\left(\sigma_{r r}^{s} \varepsilon_{r r}^{s}+\sigma_{\theta \theta}^{s} \varepsilon_{\theta \theta}^{s}+2 \sigma_{r z}^{s} \varepsilon_{r z}^{s}\right) d \Gamma$

where $V$ and $\Gamma$ are the bulk volume and the surface area of the plate respectively.

Finally, the potential energy due to a vertical load $q(r)$ as shown in Figure 1 is written as,

$W=-\int_{\Gamma} q(r) w(r) d \Gamma$

In view of Eqs. (8) to (10), the total potential energy $I_{P}$ of nano-plate is given by,

$I_{P}=U_{B}+U_{S}+W$

The minimization of $I_{P}$ in Eq. (11) together with integration by parts leads to the governing equation for a circular nano-plate:

$$
D \nabla^{4} w-2 \tau_{P}^{s} \nabla^{2} w-q(r)=0
$$

where

$$
D=\frac{E h^{3}}{12\left(1-v^{2}\right)}+\left(\lambda_{P}^{s}+2 \mu_{P}^{s}\right) \frac{h^{2}}{2}-\frac{\tau_{P}^{s} v h^{2}}{6(1-v)}
$$

and

$$
\nabla^{2}=\frac{1}{r} \frac{d}{d r}\left(r \frac{d}{d r}\right)
$$


The shear force and bending moment of a circular nano-plate are given by,

$Q_{r}=-D \frac{d}{d r} \nabla^{2} w+2 \tau_{P}^{s} \frac{d w}{d r}$

$M_{r}=D \frac{d^{2} w}{d r^{2}}+\frac{D_{1}}{r} \frac{d w}{d r}$

where

$$
D_{1}=\frac{v E h^{3}}{12\left(1-v^{2}\right)}+\left(\lambda_{P}^{s}+\tau_{P}^{s}\right) \frac{h^{2}}{2}-\frac{\tau_{P}^{s} v h^{2}}{6(1-v)}
$$

Equations (12) to (17) are identical to the governing equation for a nano-plate derived by Liu and Rajapakse [8] from the equilibrium of an infinitesimal plate element. This equation reduces to the classical Krichhoff equation when the surface energy effects is completely neglected (i.e., $\tau_{P}^{s}, \lambda_{P}^{s}, \mu_{P}^{s}$ are zero). In addition, $\lambda_{P}^{s}$ and $\mu_{P}^{s}$ are surface Lamé constants for the nano-plate.

\subsection{Strain energy of elastic half-space}

An elastic circular nano-plate under axisymmetric vertical loading is resisted by the normal contact traction acting on bottom surface of the plate. Let $S$ denote the circular contact area between the plate and the supporting medium. The normal traction can be represented by a traction field $T_{z}(r)$ acting on the circular surface $S$. The strain energy $U_{h}$ of the elastic half-space can be expressed in the following form:

$U_{h}=\frac{1}{2} \int_{0}^{a}\left[2 \pi r T_{z}(r) w(r)\right] d r$

Note that $T_{z}(r)$ can be expressed in term of generalized coordinates $\alpha_{n}$ as follows:

$T_{z}(r)=\sum_{n=0}^{N} \alpha_{n} T_{n z}(r)$

where $T_{n z}(r)$ denotes the traction in the vertical direction applied over $S$ such that the vertical displacement within $S$ is equal to $r^{2 n}$.

To determine the traction $T_{z}(r)$, the circular contact area $S$ is discretized into a number of $\mathrm{Ne}$ annular ring elements. It is assumed that $T_{n z}(r)$ is constant within each ring element. The unknown contact traction $T_{n z}(r)$ is then evaluated by solving a flexibility equation based on the Green's functions of an elastic half-space with consideration of surface energy effects under axisymmetric vertical loading. These Green's functions, expressed in the form of Hankel integral transform, are given elsewhere [14].

In view of Eqs. (11) and (18), the total potential energy $I$ of the plate-half-space system can be expressed as,
$I=I_{P}+U_{h}$

Finally, the generalized coordinates $\alpha_{n}(n=0,1, \ldots, N)$ are determined by using the principle of minimum potential energy, which requires that

$$
\frac{\partial I}{\partial \alpha_{n}}=0, \quad n=0,1, \ldots, N
$$

The solution of a linear simultaneous equation system given by Eq. (21) yields the solution of the generalized coordinates $\alpha_{n}(n=0,1, \ldots, N)$ for a given plate-half-space system. Finally, the plate deflection, shear force, and bending moment can be obtained by back substituting the generalized coordinates into Eqs. (4), (15), and (16) respectively.

\section{Numerical results and Discussion}

\subsection{Validation of numerical scheme}

The accuracy of the present solution scheme is confirmed by comparing with existing solutions. Figure 2 shows the comparison of normalized deflection profiles of a circular nanoplate with a simply-supported edge under a uniform loading, $q_{0}$, between the present solution and the solution given by Liu and Rajapakse [8]. The material parameters employed in the comparison are $E=90 \mathrm{GPa}$ and $v=0.23$; and the surface material properties are $\tau_{P}^{s}=0.5689 \mathrm{~N} / \mathrm{m}$, $\lambda_{P}^{s}=3.4939 \mathrm{~N} / \mathrm{m}$ and $\mu_{P}^{s}=-5.4251 \mathrm{~N} / \mathrm{m}$. It is clearly seen that very good agreement between the two solutions is obtained with $N=5$. Figure 3 (a) and (b) respectively show radial profiles of normalized contact traction and bending moment of a circular plate on an isotropic elastic half-space without surface stress effects, between the present solution with $N=8$ and $N e=40$ and the solution proposed by Brown [17] for different values of relative plate stiffness, $K_{r}$, with $v=0.3$. It is evident from Figure 3 that the present solution agrees very closely with Brown [17] for all values of $K_{r}$.

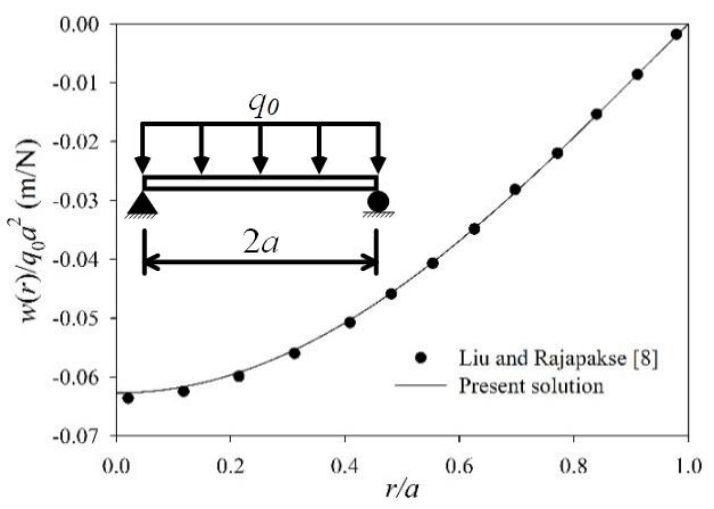

Figure 2. Comparison for deflection of a circular nano-plate. 

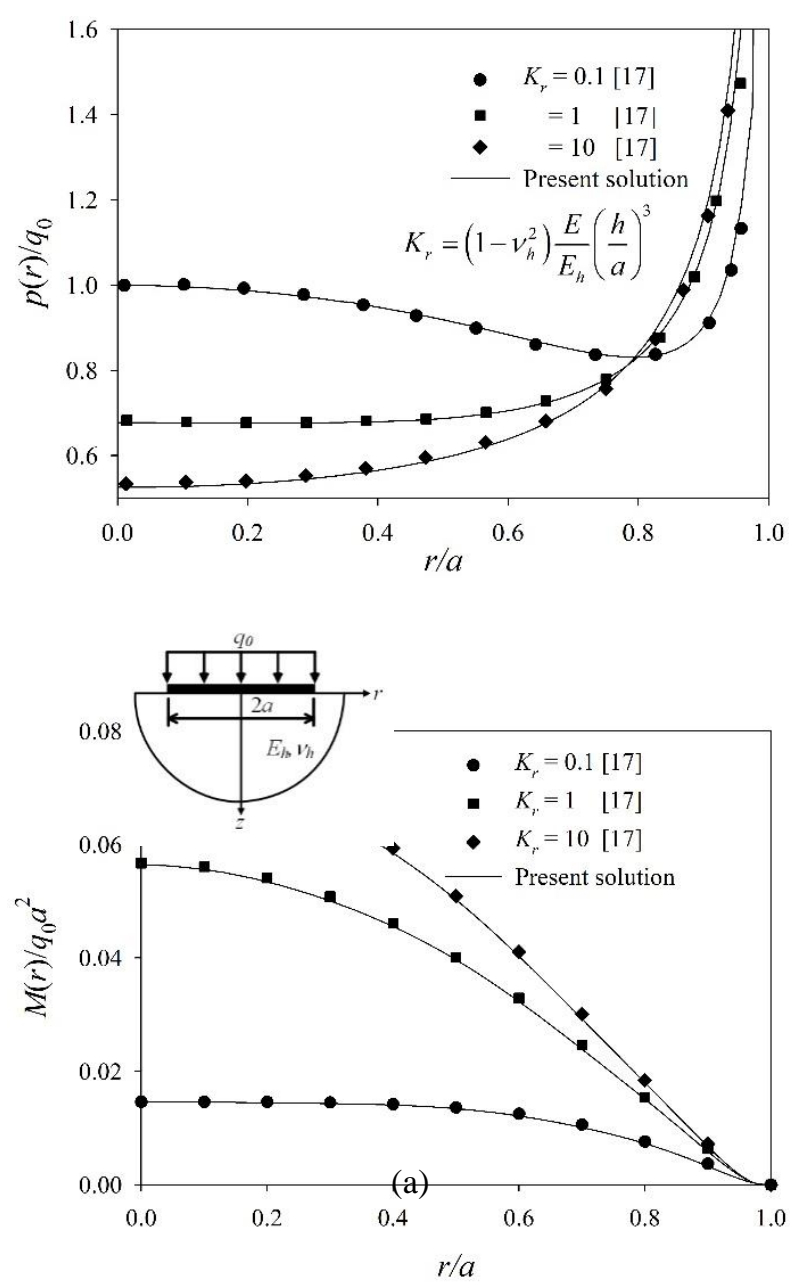

Figure 3. Comparison for: (a) contact pressure; and (b) bending moment of a circular plate on an elastic half-space without surface energy effects

\subsection{Numerical results}

The influence of surface energy effects on interaction between an elastic circular plate under axisymmetric vertical loading and an elastic half-space is investigated in Figures 4 to 6 . The following material parameters are employed in the numerical study: $E=107 \mathrm{GPa}$ and $v=$ 0.33 for the plate; and $\lambda=58.17 \mathrm{GPa}$ and $\mu=26.13 \mathrm{GPa}$ for the half-space. In addition, $\tau_{P}^{s}=0.6056 \mathrm{~N} / \mathrm{m}, \lambda_{P}^{s}=$ $4.4939 \mathrm{~N} / \mathrm{m}$, and $\mu_{P}^{s}=2.7779 \mathrm{~N} / \mathrm{m}$ for the surface of the plate; and $\tau_{h}^{s}=1 \mathrm{~N} / \mathrm{m}, \lambda_{h}^{s}=6.8511 \mathrm{~N} / \mathrm{m}$, and $\mu_{h}^{s}=-$ $0.376 \mathrm{~N} / \mathrm{m}$ for the top surface of the half-space. Note that the broken lines in all figures presented in this section denote the classical solutions where surface stress effects are ignored (i.e. $\tau^{s}=\lambda^{s}=\mu^{s} \approx 0$ ). Figure 4 and 5 respectively display radial profiles of normalized vertical displacements and bending moments of a circular nanoplate resting on an elastic half-space under a uniformly distributed vertical load of magnitude $q_{0}$ for different values of relative plate stiffness, i.e., $K_{r}=0.1,1$ and 10 . In addition, $\bar{a}=10$ where $\bar{a}=a / \Lambda_{h}$, and $\Lambda_{h}=$ $\left(\lambda_{h}^{s}+2 \mu_{h}^{s}\right)(\lambda+2 \mu) / 2 \mu(\lambda+\mu)$. Numerical results in Figure 4 and 5 indicate a significant influence of surface energy effects on both deflection and bending moment. It can be seen from Figure 4 that the normalized deflection from the present study is lower than its classical counterpart for all values of relative stiffness $K_{r}$ whereas the normalized bending moment under the influence of surface stresses shown in Figure 5 is always larger than the corresponding classical solution. Therefore, the presence of surface stresses renders the plate stiffer.

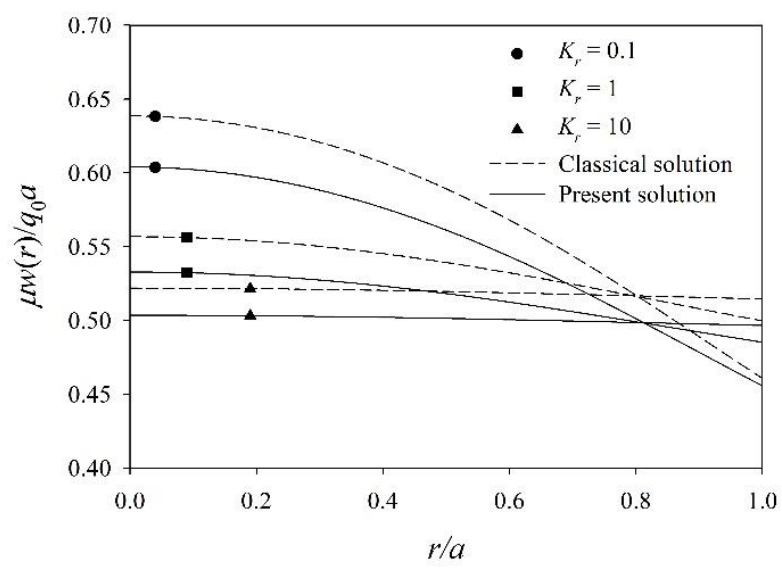

Figure 4. Radial profiles of normalized vertical deflection of a circular nano-plate resting on an elastic half-space under uniform vertical loading for different values of $K_{r}$ with $\bar{a}=10$.

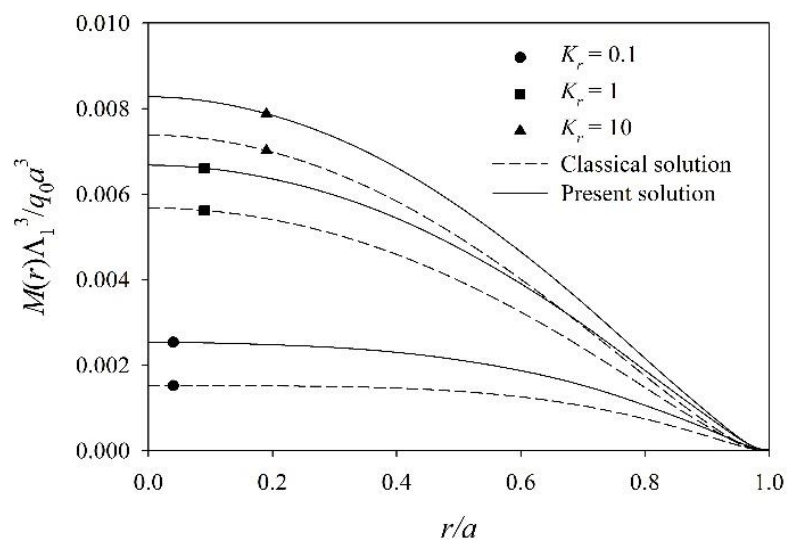

Figure 5. Radial profiles of normalized bending moment of a circular nano-plate resting on an elastic half-space under uniform vertical loading for different values of $K_{r}$ with $\bar{a}=10$.

Figure 6 shows variations of normalized central deflection of a circular nano-plate under the uniform vertical loading with normalized radius $\bar{a}$ for different values of relative stiffness $K_{r}$. It is evident from Figure 6 that under the influence of surface energy the normalized central deflection depends significantly on both normalized radius and relative stiffness of the plate. Sizedependent behaviour due to the presence of surface stresses is thus clearly observed in the present solution whereas the classical elasticity solution is sizeindependent. In addition, the influence of surface energy effects is reduced with increasing value of $\bar{a}$, and the present solution eventually converges to the classical solution. 


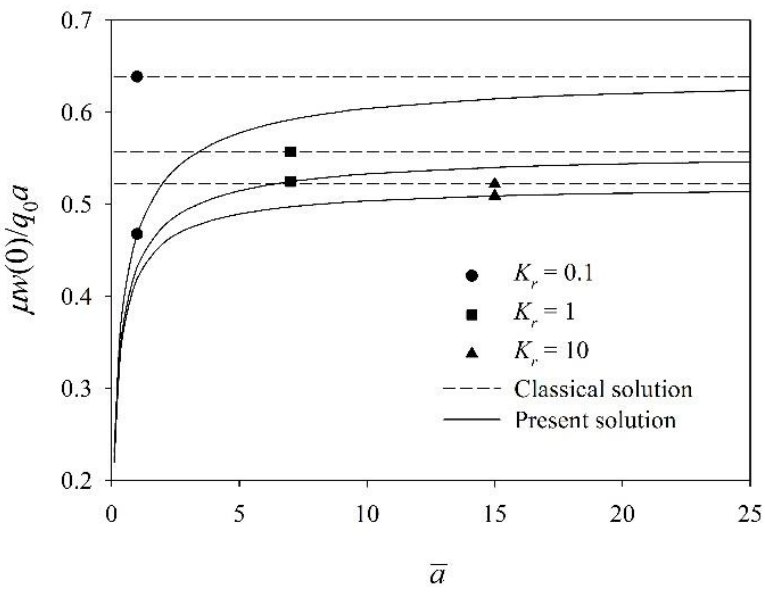

Figure 6. Variations of normalized central deflection with $\bar{a}$ of a circular nano-plate resting on an elastic half-space uniform vertical loading for different values of $K_{r}$.

\section{Conclusions}

A variational solution scheme is presented in this paper to study the interaction problem between an elastic circular plate and an isotropic elastic half-space under the influence of surface energy by adopting Gurtin-Murdoch surface elasticity theory. The contact surface between the plate and the half-space is assumed to be smooth, and the deflection shape of the plate is represented by a power series of the radial coordinate. The generalized coordinates in the series are obtained from the minimization of the total potential energy functional of the plate half-space system, which consists of the strain energies of the plate, the half-space, and the surface, together with the potential due to the applied loading. The accuracy of the proposed solution scheme is confirmed by comparing with exiting solutions. Numerical results presented in this paper indicate a significant influence of surface energy effects on the interaction problem, and the consideration of surface stresses renders the plate stiffer. In addition, the material behaviour becomes sizedependent when the surface stresses are taken into account. The proposed variational solution scheme can be used to study various interaction problems involving an elastic circular plate and an elastic medium with the presence of surface stresses for the applications related to nano-scale systems and soft elastic solids.

\section{Acknowledgement}

The work presented in this paper was supported by the Thailand Research Fund [grant numbers BRG 5880017 and PHD/0096/2554]. This support is gratefully acknowledged.

\section{References}

1. Gurtin M E and Murdoch A I 1975 Arch. Ration. Mech. An. 57 291-323.

2. Gurtin M E and Murdoch A I 1978 Int. J. Solids Struct. 14 431-440.

3. Shenoy V B 2005 Phys. Rev. B 71094104.

4. He L H and Lim C W 2006 Int. J. Solids Struct. 43 132- 143.

5. Lü C F, Lim C W and Chen W Q 2009 Int. J. Solids Struct. 46 1176-1185.

6. Intarit P, Senjuntichai $\mathrm{T}$ and Rajapakse R K N D 2010 Eng. Fract. Mech. 77 3592-3603.

7. Sapsathiarn Y and Rajapakse R K N D 2012 IEEE T Nanotechnol. 11 247-254.

8. Liu C and Rajapakse R K N D 2013 IEEE T. Nanotechnol. 12 13-20.

9. Pinyochotiwong Y, Rungamornrat $\mathbf{J}$ and Senjuntichai T 2013 Int. J. Eng. Sci. 71 15-35.

10. Rungamornrat J, Tuttipongsawat $P$ and Senjuntichai T 2016 App. Math. Model. 40 1532-1553.

11. Intarit $P$, Senjuntichai $T$, Rungamornrat $J$ and Rajapakse R K N D 2017 Acta Mech. 228 617-630

12. Tirapat S, Senjuntichai T and Rungamornrat J 2017 Adv. Mater. Sci. Eng. 20177530936

13. Intarit P, Senjuntichai T and Rungamornrat J 2018 Z. Angew. Math. Phys. 6929

14. Intarit P 2012 Solutions of Elastic Medium with Surface Stress Effects. Ph.D. Thesis, Chulalongkorn University

15. Lim C W and He L H 2004 Int. J. Mech. Sci. 46 $1715-1726$.

16. Lu P, He L H, Lee H P and Lu C 2006 Int. J. Solid Struc. 43 4631-4647.

17. Brown 1969 Geotechnique 19 399-404 